\title{
The quasi-biennial periodicity (QBP) in velocity and intensity helioseismic observations
}

\section{The seismic QBP over solar cycle 23}

\author{
R. Simoniello ${ }^{1}$, W. Finsterle ${ }^{1}$, D. Salabert ${ }^{2}$, R. A. García ${ }^{3}$, S. Turck-Chièze ${ }^{3}$, A. Jiménez ${ }^{4}$, and M. Roth ${ }^{5}$ \\ ${ }^{1}$ PMOD/WRC Physikalisch-Meteorologisches Observatorium Davos-World Radiation Center, 7260 Davos Dorf, Switzerland \\ e-mail: [rosaria.simoniello; wolfgang. finsterle]@pmodwrc.ch \\ ${ }^{2}$ Université de Nice Sophia-Antipolis, CNRS UMR 6202, Observatoire de la Côte d'Azur, BP 4229, 06304 Nice Cedex 4, France \\ e-mail: salabert.david@oca.fr \\ ${ }^{3}$ Laboratoire AIM, CEA/DSM-CNRS-Université Paris Diderot, CEA, IRFU, SAp, centre de Saclay, 91191 Gif-sur-Yvette, France \\ e-mail: [rgarcia;sylvaine.turck-chieze]@cea.fr \\ ${ }^{4}$ IAC, Instituto de Astrofisíca de Canarias, 38205 La Laguna, Tenerife, Spain \\ e-mail: ajm@iac.es \\ 5 Kiepenheuer Institute for Solar Physics, Freiburg, Germany \\ e-mail: mroth@kis.uni-freiburg.de
}

Received 9 September 2011 / Accepted 12 January 2012

\begin{abstract}
Aims. We looked for signatures of quasi-biennial periodicity (QBP) over different phases of solar cycle by means of acoustic modes of oscillation. Low-degree $p$-mode frequencies are shown to be sensitive to changes in magnetic activity due to the global dynamo. Recently there has been reported evidence of two-year variations in $p$-mode frequencies.

Methods. Long high-quality helioseismic data are provided by BiSON (Birmingham Solar Oscillation Network), GONG (Global Oscillation Network Group), GOLF (Global Oscillation at Low Frequency) and VIRGO (Variability of Solar IRradiance and Gravity Oscillation) instruments. We determined the solar cycle changes in $p$-mode frequencies for spherical degree $\ell=0,1,2$ with their azimuthal components in the frequency range $2.5 \mathrm{mHz} \leq v \leq 3.5 \mathrm{mHz}$.

Results. We found signatures of QBP at all levels of solar activity in the modes more sensitive to higher latitudes. The signal strength increases with latitude and the equatorial component also seems to be modulated by the 11-year envelope.

Conclusions. The persistent nature of the seismic QBP is not observed in the surface activity indices, where mid-term variations are found only from time to time and mainly in periods of high activity. This feature, together with the latitudinal dependence, provides more evidence of a mechanism that is almost independent and different from the one that brings the active regions up to the surface. Therefore, these findings can be used to provide more constraints on dynamo models that consider a further cyclic component on top of the 11-year cycle.
\end{abstract}

Key words. methods: data analysis - Sun: helioseismology - Sun: activity

\section{Introduction}

Evidence of shorter periodicities than the 11-year period in solar activity proxies, ranging from days to years, are very well known in the literature. For short terms, the Sun exhibits 27-day and 13.5-day periodicities, while the regime between days and 11 years are referred to as mid-term periodicities (Bai 2003). Particularly prominent are the ones between 1.5 and 4 years (Vecchio et al. 2009) and hereafter these are referred to as quasibiennial periodicity (QBP). This two-year cycle has been observed in radio flux (RF) on $10.7 \mathrm{~cm}$ (Belmont et al. 1996), total solar irradiance (Penza et al. 2006), flares and sunspots (Akioka 1987; Mursula et al. 2003; Valdés-Galicia \& Velasco 2008), and a green coronal emission line (Vecchio \& Carbone 2008; Vecchio et al. 2009). In these activity proxies the QBP appears only time to time and tends to be stronger over periods coinciding with solar maxima, and they are not recognized as typical feature of every cycle (Krivova \& Solanki 2002; Knaack et al. 2004; Cadavid et al. 2005; Li et al. 2006). This suggests that the mechanism behind the QBP might be closely linked to the strength of the global dynamo. Coronal data have shown a latitudinal dependence on the signal strength (Vecchio \& Carbone 2008). It is particularly strong in the polar region and weaker around the equator, and moreover, the periodicity from northern and equatorial regions $(\approx 2.8$ years $)$ is different from the one in southern regions $(\approx 1.5$ years). More recently the same authors have investigated a longer dataset recorded from 1939 to 2005 and concluded that the non-constant period lengths is the manifestation of a unique quasi-biennial activity cycle (Vecchio et al. 2009).

Since the discovery of the correlation between the observed global acoustic mode ( $p$-mode) parameters and the solar magnetic activity cycle (e.g. Woodard \& Noyes 1985; Pallé et al. 1989; Elsworth et al. 1993), it became evident that the acoustic modes can also be used as a diagnostic tool of the solar cycle. In particular, with high-quality and continuous helioseismic data, deeper analysis has revealed that mode amplitudes are suppressed with increasing magnetic activity, while mode frequencies are shifted towards higher values as the solar cycle approaches its maximum at low and high-angular degrees 
(Howe et al. 1999; Komm et al. 2000; Jiménez-Reyes et al. 2001; Chaplin et al. 2001; Salabert et al. 2004; Salabert \& Jiménez-Reyes 2006; Simoniello et al. 2010). While a possible explanation in terms of mode conversion has been provided for the mode amplitudes (Simoniello et al. 2010), we still lack the basic understanding of the mode frequency shift. $F$-modes can help for understanding this variability and the comparison of observations to predictions seem to show some structural effects (Lefebvre et al. 2009). Unfortunately, a proper introduction of subsurface magnetic field is still lacking, so this does not allow us to properly estimate the structural consequences of this important factor. The form and the degree dependence of the shifts favor near-subsurface phenomenon, but they cannot be explained entirely by structural changes, since the magnetic field is somehow involved in the mechanism (Goode \& Dziembowski 2002; Dziembowski \& Goode 2004). Recently, the argument that the solar cycle variation in the global mode frequency is due to the global averaging of active regions (Hindman et al. 2001) has only been partially supported by the observations (Tripathy et al. 2010). It has been argued that the weak component of the magnetic field (turbulent or horizontal field) must be taken into account in order to fully explain the observed mode frequency shift.

Recent observations in $p$-mode frequencies have also shown the presence of a two-year modulation most prominent over periods coinciding with solar maxima. The properties of the seismic QBP have been interpreted as the visible manifestation of a second dynamo mechanism (Benevolenskaja 1995, 1998a,b), which is induced by the strong latitudinal shear located below the surface at $\approx 0.95$ solar radius (Schou et al. 1998). The second dynamo itself cannot explain the observed modulation in the signal strength, and therefore it has been supposed that, when the 11-year cycle is in its strong phase, buoyant magnetic flux is sent upward by the main dynamo into shallower layers, thus allowing the seismic QBP to be mainly detected over periods of solar maxima (Fletcher et al. 2010). However, it is still a matter of debate whether this second dynamo mechanism is indeed behind the QBP observed in activity proxies and in solar seismic data. In fact, recent observations of rapidly rotator stars have shown signatures of QBP, whose strength increases with increasing magnetic activity, and it gets stronger at polar regions. It has been supposed that the unstable magnetic Rossby waves in the tachocline might be behind it (Zaqarashvili et al. 2010, 2011). Whether this is also true for solar type stars is still an ongoing investigation.

The $p$-mode spatial configuration can be described by spherical harmonics with each mode characterized by its spherical harmonic degree $\ell$ and azimuthal order $m$. Modes with $\ell \leq 3$ are named low-degree modes and conversely to surface activity indices, they also sound the interior of the Sun being shaped in deeper layers. Therefore these modes might feel magnetic changes that have yet to show up at the surface, or it might also be possible that the disturbances will never reach the Sun's surface.

By averaging the individual $p$-mode frequency shifts over all low $(\ell)$-values, observational findings pointed to seismic signatures of QBP over the long extended minimum characterizing the end of solar cycle 23 and beginning of solar cycle 24 (Broomhall et al. 2009a; Fletcher et al. 2010). Further evidence of this feature has been reported in low-degree modes by investigating the spherical degree dependence (Broomhall et al. 2011), but some authors have cast doubts, because they did not spot any significant trend during periods of low activity (Jain et al. 2011).
We decided to investigate the properties of the seismic QBP by using several helioseismic instruments in velocity and intensity data covering a period that goes from the end of solar cycle 22 up to the beginning of solar cycle 24. Each observational program can provide more stable and/or accurate data depending on instrumental features (Broomhall et al. 2009b; Salabert et al. 2011) and therefore, by comparing different observations, we have been able to check whether or not the seismic QBP occurs only time to time and during periods of high activity.

We analyzed the $p$-mode frequency shifts for each of the sectoral $(m=\ell)$ and zonal $(m=0)$ components of low-degree modes (without averaging over the azimuthal components) and this is the novelty of the work within this topic. We also investigated to what extent the mid-term periodicity is linked to the appearance of active regions induced by the main dynamo. To this aim we carried out a correlation analysis between each azimuthal ( $m$ )-components of low-degree mode frequencies and activity indices linked to strong and/or weak magnetic fields over different phases of solar cycle 23. It is important to investigate the phasewise variation in correlation between low- $\ell$ frequencies and activity indices as well, because earlier study of the $m$-components were confined to intermediate degree modes (Jain et al. 2009). Furthermore, acoustic waves, depending on their spherical degree and azimuthal component, are more sensitive to lower or to higher latitudes. Therefore the analysis of the azimuthal components also allowed us to have some hints on the role played by strong and/or weak fields with latitudes as sources of the QBP.

The seismic investigation of the mid-term periodicity might help us gain a deeper insight into the global properties of the Sun such as on the solar dynamo theory or on stellar cycles: mid-term periodicities have already been observed in fast-rotator stars and therefore the better understanding of this short periodic behavior in the Sun might help illuminate the interpretation of this signal of solar-like stars.

\section{Helioseismic data analysis}

\subsection{Mode visibility}

Low-degree modes are detected either in Sun-as-a-star observations or through highly spatially resolved images. In the first scenario the modes are observed in averages made over the visible disk of Doppler velocity or intensity variations. In this case only the modes that satisfy the condition $\ell+m=$ even are visible, because of the near-perpendicular inclination of the Sun with respect to the Earth. In observations with high spatial resolution, the visible disk is sampled in many pixels and the collected images are decomposed into their constituent spherical harmonics, giving access to all $2 \ell+1$ component for each degree.

\subsection{Sun-as-a-star observations from ground based network}

Birmingham Solar Oscillation Network (BiSON) has been operating since 1976 and makes unresolved solar disk observations. It consists of six stations using resonant scattering spectrometers that perform Doppler velocity measurements in integrated sunlight of the K Fraunhofer line at $7699 \AA$ (Chaplin et al. 1995). The data are dominated by the Doppler variations from the low-degree, $\ell$, modes. The nominal height of observation is at $\approx 260 \mathrm{~km}$ above the photosphere (e.g. Jiménez-Reyes et al. 2007). We analyzed the data provided by BiSON instruments from 11 April 1996 to 4 April 2009. This long dataset was split into contiguous 365-day series with four overlaps of 91.25 days. Estimates of the $p$-mode frequencies for $\ell=0,1,2$ 
were extracted from each subset by fitting a modified Lorentzian model to the data using a standard likelihood maximization method (Fletcher et al. 2009).

\subsection{Sun-as-a-star observations from space}

The Global Oscillation at Low Frequency (GOLF) instrument, onboard the SOlar and Heliospheric Observatory (SOHO) satellite, is devoted to the search for low-degree modes (Gabriel et al. 1995). It works by measuring the Doppler shifts of the Na line at $5889 \AA$ (D1) and $5896 \AA$ (D2). Owing to the malfunctioning of the polarization system, GOLF works in a single-wing configuration, and it has been proven that it is almost a pure velocity signal (Pallé et al. 1999). The one-wing configuration has been changing during the 14 years of observations as follows: 1) 1996-1998 in the blue-wing configuration; 2) 1998-2002 in the red-wing configuration; 3 ) up to today in the blue-wing configuration (Ulrich et al. 2000; García et al. 2005).

Due to the formation heights of the spectral lines, in the blue-wing configuration, GOLF observes at $\approx 330 \mathrm{~km}$, while in the red-wing configuration at $\approx 480 \mathrm{~km}$ (Jiménez-Reyes et al. 2007). Although the changing observational height affects the p-mode amplitude determination (Simoniello \& Salabert 2009; Simoniello et al. 2010), it does not affect the $p$-mode frequency. We analyzed the data provided by GOLF instruments from 11 April 1996 to 4 of April 2009 and the estimates of $p$-mode frequency shifts were extracted by applying the same fitting method as in BiSON. This differs from the one applied to VIRGO and GONG $\ell=0$ integrated. We, therefore, verified that both fittings methods returned consistent results.

The Solar PhotoMeters (SPM) of the Variability of solar IRradiance and Gravity Oscillations (VIRGO) package (Fröhlich et al. 1995, 1997) onboard the SOHO satellite consists of three independent photometers, centered around 402, 500 and $862 \mathrm{~nm}$ (the blue, green, and red channels respectively). They measure the spatially integrated solar intensity over a $5 \mathrm{~nm}$ bandpass at a one minute cadence. The data obtained by the VIRGO/SPM instrument over the mission have been of uniformly high quality, whether before or after the loss of contact with the spacecraft for several months in 1998. Each one of the three different signals observes the Sun-as-a-star oscillations at different heights in the solar atmosphere (Jiménez et al. 2005).

The data provided by VIRGO instruments cover almost 14 years of data, starting on 11 April 1996 up to 8 April 2010. This dataset was split into contiguous 365-day with four overlaps of 91.25 days. The power spectrum of each subseries was fitted to estimate the mode $p$-mode parameters for $\ell=0,1,2$ using a standard likelihood maximization function (Salabert et al. 2007). Each mode component is parameterized using a asymmetric Lorentzian profile.

\subsection{High-resolved observations from ground based network}

The Global Oscillation Network Group (GONG) consist of six instruments deployed worldwide to provide nearly continuous and stable velocity images of the Sun. GONG instrument is based on a Michelson interferometer called a Fourier tachometer (Beckers \& Brown 1978). It works by using the Ni line at $676.8 \mathrm{~nm}$, and the observational height is approximately at $213 \mathrm{~km}$. The network started to be fully operating since May 1995. Time series of 36 days (the so-called GONG month) are produced, and the individual frequencies of the azimuthal components are determined up to $\ell=150$ over subseries of 108 days
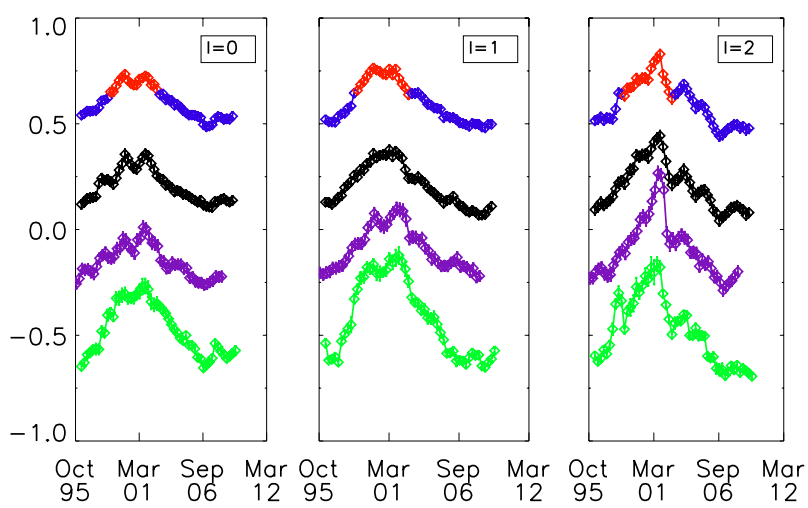

Fig. 1. Solar cycle changes in $p$-mode frequency shifts $(\mu \mathrm{Hz})$ for $\ell=$ $0,1,2$ from VIRGO (green), BiSON (black), GONG (purple), and GOLF (blue and red to identify the corresponding wing configuration) observations over solar cycle 23 and beginning of solar cycle 24 .

by concatenating three GONG months. These frequencies are afterwards made publicly available (http://gong2.nso.edu/ archive/).

The $\ell=0$ GONG time series between 7 May 1995 and 3 May 2008 was analyzed in a similar manner to VIRGO data, returning estimates of the mode frequencies of $\ell=0,1,2$. To analyze the azimuthal components individually, we made use of the frequency tables of the $\ell=1,2$ modes available from the GONG webpage between 29 of June 1995 and 24 December 2009. Very few outliers were removed from the following analysis using the quality flags provided by the GONG team.

\subsection{Frequency shift determination}

The temporal variations of the $p$-mode frequencies were defined as the difference between the frequencies of the corresponding modes observed on different dates and reference values taken as the average over the years 1996-1997. The weighted averages of these frequency shifts were then calculated between 2500 and $3500 \mu \mathrm{Hz}$. Furthermore, for each spherical degree the shifts from the VIRGO green, blue, and red channels were averaged. The shifts for each spherical degree with the same $|\mathrm{m}|$ obtained by the azimuthal analysis of GONG high-resolved data were also averaged.

\section{QBP signatures in $p$-mode frequencies over solar cycle 23}

\subsection{Spherical degree dependence of the QBP over the 11-year cycle}

We looked for QBP signatures in $p$-mode frequency shifts over the 11-year cycle. In Fig. 1 we show the spherical degree dependence of the shift as seen from VIRGO, BiSON, GONG, and GOLF datasets. We introduced an artificial offset between the curves to better compare the seismic features. We can spot several seismic signatures that might be linked to the QBP signal. For $\ell=0$ modes over the minimum of solar cycle 22 and beginning of solar cycle 23 (1995-1997), GONG observations show the seismic signature of a QBP. Following the ascending phase of solar cycle 23 in BiSON and GONG, between 1997-1999, we find a further modulation of about two years. This signature does not appear in GOLF and VIRGO data, probably due to the loss of SOHO in June 1998. During the maximum of solar activity the modes show up with a clear double-peak structure in all datasets. 

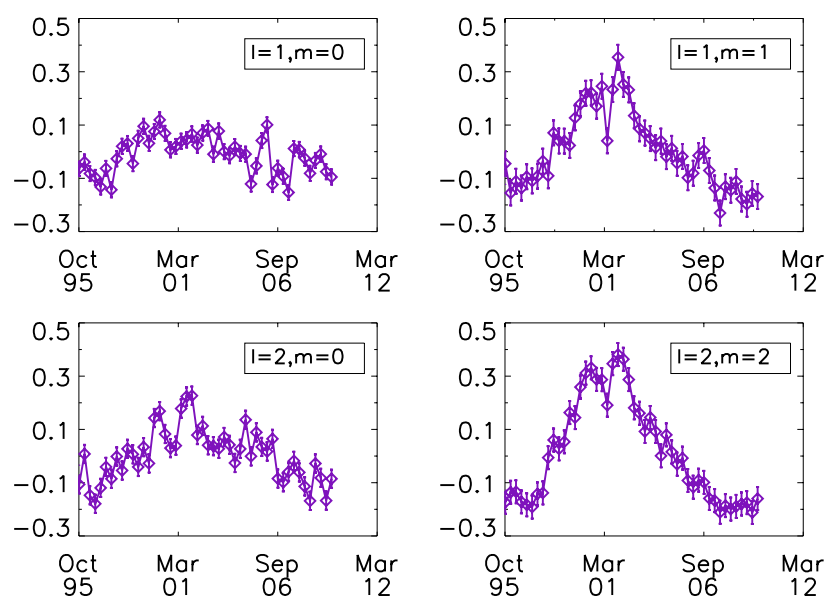

Fig. 2. Solar cycle changes in $p$-mode frequency shifts $(\mu \mathrm{Hz})$ for the $m$-components of $\ell=1,2$ modes as seen from GONG observations.

Over the descending phase BiSON, GOLF, and VIRGO agree quite well, showing the same downward trend not characterized by any modulation. GONG data, instead, shows an oscillatory signal after March 2001. Towards the end of solar cycle 23 and beginning of solar cycle 24 (after September 2006), we observe a QBP in all datasets although in GOLF and VIRGO data are particularly prominent. In $\ell=1$ modes there are no traces of QBP over the ascending phase in BiSON, GOLF, and VIRGO data. Instead GONG shows a modulation around July 1998. Over the maximum phase we found a clear double-peak structure in all datasets, but in GONG it is more pronounced. In GONG, BiSON, and GOLF, over the descending phase, we might spot two further modulations soon after March 2001 and before September 2006 that are instead absent in VIRGO data. Over the long extended minimum of solar cycle 23 , only VIRGO shows a further two-year modulation after September 2006. In $\ell=2$ modes we found over the minimum of solar cycle 22 and beginning of solar cycle 23 (1995-1997) a seismic signature of a QBP from GONG observations. We found the same signature in $\ell=0$ modes in GONG data. Over the ascending phase, a strong peak of an almost two-year length around 1998 in VIRGO and GOLF data is present. This signal might be an artefact owing to the loss of the satellite between June and September 1998. Over the maximum phase we found a double-peak structure in BiSON, GONG, and GOLF observations. The descending phase is characterized by a very well-modulated signal of about two years, although it is more pronounced in BiSON, GOLF, and VIRGO. During the minimum of solar cycle 23, we spotted in BiSON and GOLF data a clear signature of a further periodicity starting in September 2006 and ending before June 2009.

In summary we found signatures of biennial modulation in all observational program, but there are differences in the data sets. The signal strength of mid-term variations is weaker compared to the 11-year signal. In a recent paper the mode visibility in GOLF data appears different in the two wing-configurations and it is slightly better during red-wing period (Salabert et al. 2011). This might explain why the faint signal of the QBP is less prominent in GOLF data. For intensity observations other aspects need to be taken into account, such as the selection of the spectral region in the observations that affect the mode visibility of the modes in photometric measurements (Nutto et al. 2008). Furthermore, the continuum intensity data samples a different (i.e. deeper) region where the temperature variations induced by the acoustic oscillations may be quite small.
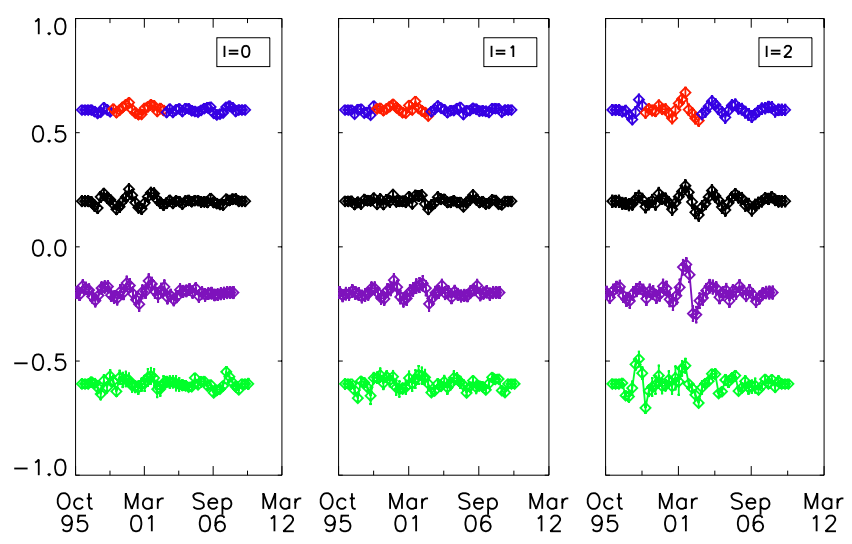

Fig. 3. The QBP signal in each low-degree $p$-mode frequency shifts after the dominant 11-yr signal has been removed. The color legend is the same as Fig. 1.

By analyzing the spherical degree dependence of $p$-mode frequency shift, we detected likely signatures of the two-year periodicity at all levels of solar activity; instead, surface activity indices mainly show it over periods coinciding with solar maxima. We also noticed that, away from the solar maximum, the QBP shows up in each low-degree mode over different periods of solar activity phase. This results from the different spatial configuration of the modes over the solar disk. For example, we found seismic signatures over the low-activity phase in the modes $\ell=0,2$ but not in the dipole mode $\ell=1$. In integrated sunlight observations due to the mode visibility, the observed $\ell=1$ frequency is a weighted measurement of the visible components $(\ell=1,|m|=1)$, while in the case of the $\ell=2$ mode, the fitted frequency corresponds to the weighted measurement of the zonal $(\ell=2, m=0)$ and sectoral $(\ell=2,|m|=2)$ components (Jiménez-Reyes et al. 2004). The spatial structure of the oscillation means that the sectoral components $|m|=\ell$ are more concentrated around equatorial latitudes than the zonal components $(m=0)$, and this is particularly true with increasing $\ell$ (Christensen-Dalsgaard 2002). As a consequence, the contribution to the QBP signal over the minimum activity might have its origin at higher latitudes (Salabert et al. 2009). Furthermore, the presence of the QBP signal over periods of low activity is not peculiar to solar cycle 23, as guessed by some authors (Fletcher et al. 2010).

\subsection{Azimuthal dependence of the QBP over the 11-year cycle}

We used GONG data to look for a QBP signal in the azimuthal components of the dipole $(\ell=1)$ and quadrupole $(\ell=2)$ mode. In Fig. 2 we show the solar cycle changes in $p$-mode frequency shifts. The sectoral components exhibit a stronger 11-year envelope than do the zonal ones. This finding clearly confirms the different sensitivity of the modes to magnetic changes with latitude. The $\ell=1, m=0$ seems to show a mid-term periodicity over the end of solar cycle 23 with an upward trend starting soon after September 2006. If we look, instead, at the $\ell=1$ $m=1$ component, we spot the double-peak structure over the maximum of solar activity as also observed in integrated sunlight observations. A further mid-term periodicity seems to occur over September 2006, and the same structure appears in GONG (integrated) and BiSON data. Looking at both $m$-components of the quadrupole mode, the solar maxima is also characterized by the typical double-peak structure. Then over the descending phase, 


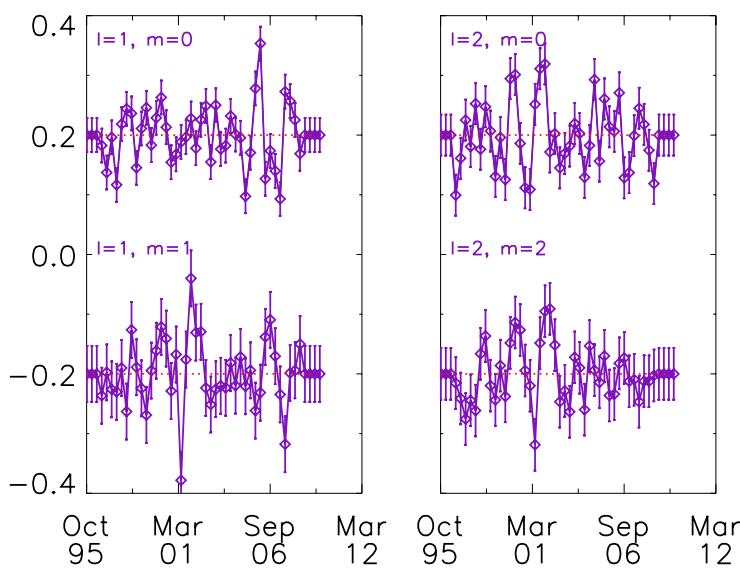

Fig. 4. The QBP signal in the zonal and sectoral component of the dipole and quadrupole mode frequency shifts after the dominant 11-yr signal has been removed from GONG high-resolved observations.

the $m=2$ component shows one smoother signature of QBP across September 2006 as in $\ell=1, m=1$. The zonal component, instead, seems to show a further periodicity starting soon after September 2006 as we already found in the mode $\ell=1$, $m=0$. Therefore the azimuthal analysis brings more evidence that the QBP might have occurred at high latitude over the minimum activity phase of solar cycle 23 .

\section{Latitudinal dependence of the QBP signal strength}

\subsection{Modulation in the QBP signal strength}

To better reveal the seismic signatures of the QBP signal, we took out the 11-year dependence by using a boxcar of 2.5 years, as already done in (Fletcher et al. 2010). In Fig. 3 we show the changes in $p$-mode frequency shifts due to the presence of the QBP signal for $\ell=0,1,2$ over solar cycle 23. GONG, BiSON, VIRGO, and GOLF observations point to a modulation of the signal strength in phase with the solar cycle in agreement with previous findings (Fletcher et al. 2010; Broomhall et al. 2011). It also seems that the modulation affects the $\ell=1,2$ modes more than $\ell=0$. This is further evidence of the higher sensitivity at low to middle latitudes of the sectoral modes. Therefore we might also argue that the observed QBP signal in the sectoral modes might come mainly from low to middle latitudes. If it is indeed the case, the signal at equatorial regions is of comparable strength (or even higher) to the QBP signal produced at high latitudes. This conclusion will not be correct at this stage. Depending on its strength and inclination (Schunker $\&$ Cally 2006), the magnetic field affects the size of the acoustic cavity, where the mode propagates. But like the acoustic cavity decreases when the spherical degree increases, therefore, to get information on the strength of the signal coming at different latitudes, we have to compare sectoral and azimuthal components of the same spherical degree. In Fig. 4 we show the QBP signal over solar cycle 23 for the $m$-components of the dipole and quadrupole modes. The residuals for $\ell=1, m=0$ might indicate the presence of a QBP across July 1998 and soon after September 2006. If we look, instead, at the $\ell=1$ $m=1$ component, we spot an increasing trend in the signal strength of the QBP over the ascending and maximum phase of solar cycle 23. A further periodicity seems to occur across September 2006, and the same structure appears in GONG (integrated) and BiSON data. Looking at the zonal component of $\ell=2$, we find over the solar maxima the typical doublepeak structure and a further mid-term periodicity soon after September 2006 as in $\ell=1, m=0$. The residuals for the sectoral component shows an increasing trend in the signal strength of the QBP over the ascending phase of solar cycle 23. A further two-year modulation might has occurred over September 2006 as found also in $\ell=1, m=1$. The azimuthal analysis confirms that the modulation in the signal strength mainly occurs in the sectoral components of the modes and also that over the long, extended minimum of solar cycle 23 the sources of the mid-term periodicity might be located at high latitudes.

\subsection{QBP signal strength over high and low activity phases}

The zonal and sectoral components of the quadrupole mode show clear signatures of the QBP signal over periods coinciding with solar maxima. By comparing the peak amplitude of the $m$-components over this activity phase, the signal seems to be of comparable strength at different latitudes. But as the strong magnetic fields are confined at low to middle latitudes, the comparison should be carried out over periods of low activity, when the magnetism of the Sun at low, as well as at high latitudes, is dominated by weak fields. To this aim we might attempt to compare the strength of the possible signatures of the QBP signal we found over the years 2006-2009. For the quadrupole mode the strength is larger for the $m=0$ compared to $m=2$ component and the same feature occurs in the dipole mode. As a consequence this might be another hint that the QBP signal at high latitudes is stronger than the one at low to middle latitudes.

\subsection{Investigating the significance of the QBP signatures over solar cycle}

Evidence of QBP has been found in all the modes. To investigate their significance, we applied the wavelet analysis developed by Torrence \& Compo (1998) to the spherical and azimuthal components of the modes. We only show the results for GONG observations, as the findings for the spherical degree analysis from the different datasets are in good agreement. In Fig. 5 we show the wavelet analysis carried out for each spherical degree. Within the cone of influence the local wavelet power spectrum identifies the QBP signal over the years 1998-2004 of solar cycle 23 in all low-degree modes. The significant periodicity at $90 \%$ of confidence level occurs at $T=1.7$ for $\ell=0,2$, while for $\ell=1$ we have two peaks above the $90 \%$ confidence level corresponding to $T=1.7$ and $T=2.1$. In Figs. 6 and 7 we show the wavelet analysis for the azimuthal components of the modes. In this case we dropped the significant level at $80 \%$ to show that there are signatures of QBP signal over the minimum activity phase in the zonal components of the modes. The significant periodicity at $80 \%$ of confidence level occurs at $T=1.7$ for $\ell=1, m=0, T=2.1$ for $\ell=1,2$ and $m=1,2$ and $T=1.6$ for $\ell=2, m=0$. It is interesting to note that the zonal modes show a slightly different periodicity from the one detected in the sectoral components. This might be the result of the greater sensitivity to higher latitudes of the zonal modes. To investigate this point further, it is important to study the properties of the QBP signal in the azimuthal components of intermediate and high-degree modes. There is evidence, in fact, that the periodicity from northern $\left(50^{\circ} \leq \theta \leq 90^{\circ}\right)$ and equatorial $\left(-45^{\circ} \leq \theta \leq 45^{\circ}\right)$ regions differs from the one in the southern regions $\left(-50^{\circ} \leq \theta \leq-90^{\circ}\right)$ (Vecchio \& Carbone 2008). 


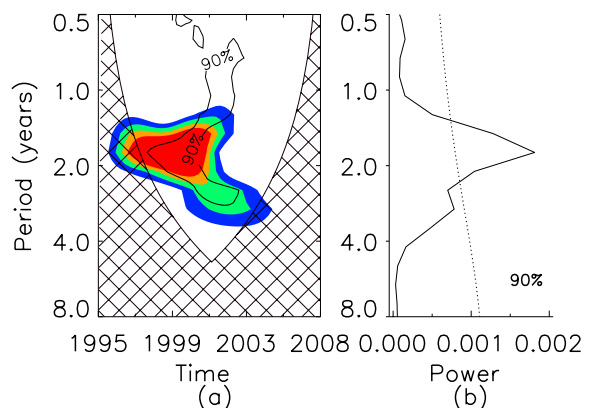

(a)

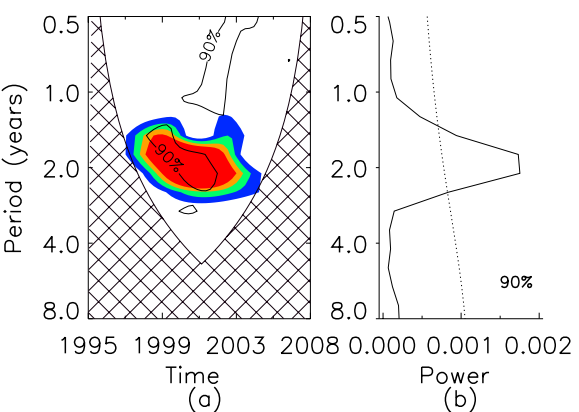

(a)

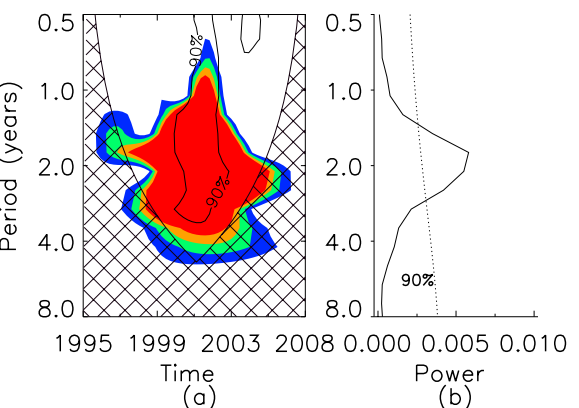

(b)

Fig. 5. a) The local wavelet power spectrum for $\ell=0,1,2$. White represent areas of little power, red those with the largest power. Solid rings identify areas at power of $0.001,0.002,0.005$ over time, and only those that achieved $90 \%$ confidence level are labeled. b) Global wavelet power spectrum. The dotted line are the $90 \%$ of confidence level.

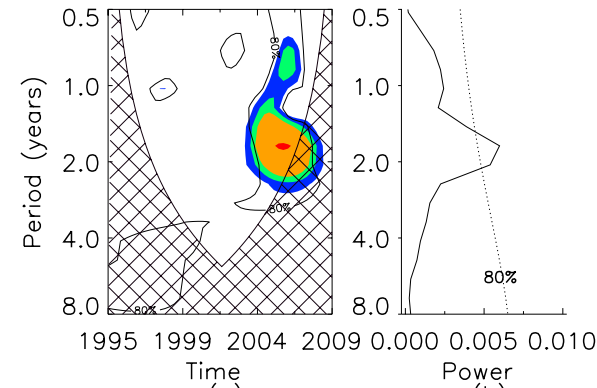

(a)

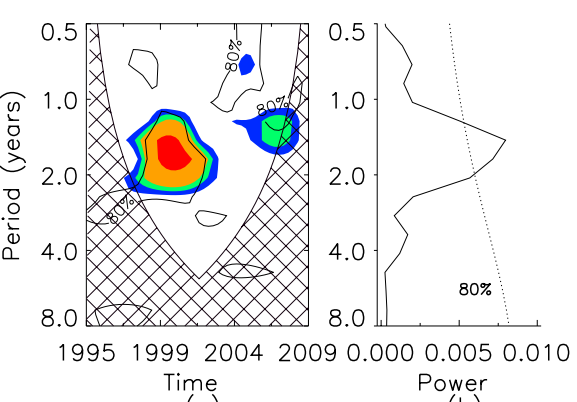

(a)

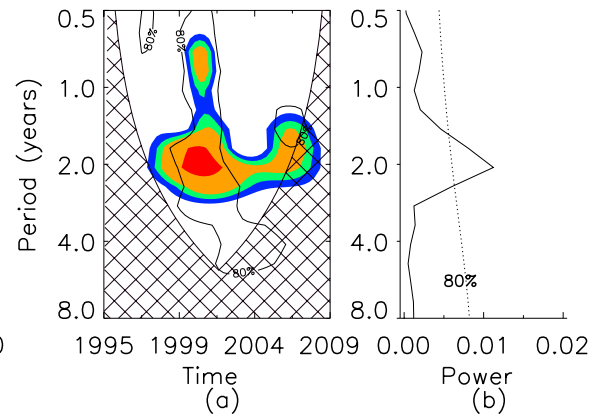

(b)
Fig. 6. The same as in Fig. 5 for the $\ell=1$ $m=0,1$. Solid rings identify areas at power of $0.005,0.0075,0.01$ over time, and only those that achieved $80 \%$ confidence level are labeled.
In summary the wavelet analysis confirms that a further periodicity of approximately two-years is indeed present in the data at confidence level of $90 \%$. Furthermore, the findings seem also to point to a likely persistent nature of the QBP signal, since we found evidence that this mid-term periodicity is also present over periods of the low-activity phase.

\section{5. $p$-mode frequency shift correlation coefficient with activity proxies over the 11-year cycle}

\subsection{Spherical degree dependence of the correlation coefficient}

The correlation analysis between the $p$-mode frequency shift and surface activity measures might improve our understanding of the role played by strong and weak fields in the QBP signal. We therefore decided to investigate the degree of correlation between $p$-mode frequency shift and two activity proxies: MWSI (Mount Wilson Sunspot Index) and F10.7 index. The MWSI values are determined as a summation over pixels where the absolute value of the magnetic field strength is greater than 100 Gauss. We have chosen MWSI because it is linked to the strong component of the toroidal fields generated by the global dynamo. These fields are mainly located at low to middle latitudes. F10.7 (RF) represents, instead, an integrated activity proxy and tells us the irradiance at $2.8 \mathrm{GHz}$ at chromospheric heights coming mainly from the Quiet Sun background emission and also sunspots and plages. This proxy therefore is a good measure of the weak (located at high latitudes) and strong magnetic fields (mainly at low to middle latitudes).

We investigated the degree of correlation of $p$-mode frequency shift with MWSI and RF by splitting the whole cycle in different phases as follows:

- AP = Ascending Phase from 22 September 1996 until 26 June 1999;

- MP = Maximum Phase 1999 June 27 to 2003 January 12;

- DP = Descending Phase January 13200326 until July 2007;

- $\mathrm{mP}=$ minimum Phase up to 2009.

We used the Spearman's rank correlation in preference to the Pearson's correlation because the Pearson's rank correlation assesses the linear correlation between two sets of data. The 

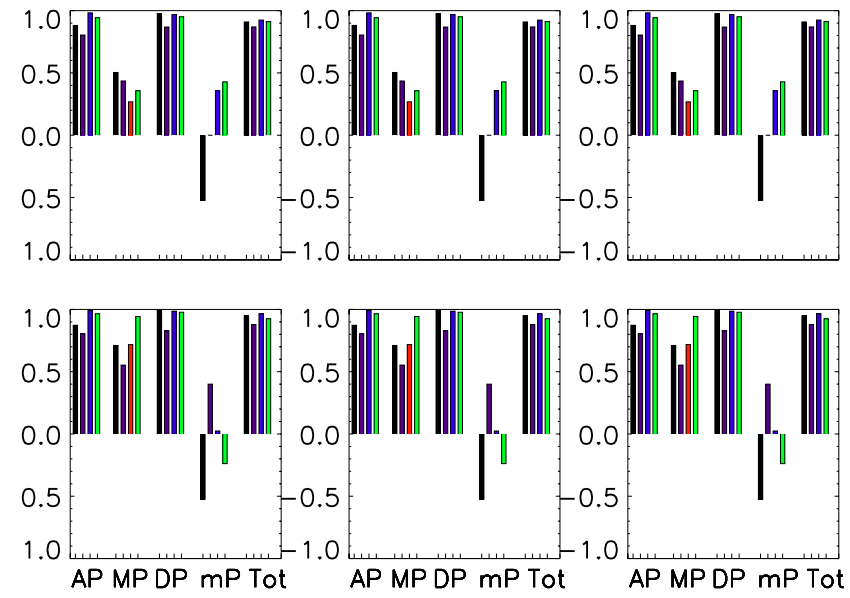

Fig. 8. The degree of correlation between $p$-mode frequency shifts of low-degree modes $(\ell=0,1,2$ from left to right) and MWSI (top panel) and RF (bottom panel) over different phases of solar activity. The bar colors identify the observational program, whose legend is the same as Fig. 1.

Spearman's rank correlation is less specific. While some of the data sets we compare may be linearly related, this is not definitely true in every case. In Fig. 8 we show the correlation coefficient for $\ell=0,1,2$ mode frequency shifts with MWSI (upper panel) and RF (bottom panel) over different phases of solar cycle for BiSON, GONG, GOLF, and VIRGO. We find that the degree of correlation of $p$-mode frequency shift with MWSI decreases over the maximum phase independently from $\ell$ value. If we look at RF we find higher correlation compared to MWSI over all the different phases of the solar cycle. These findings agree with previous results (Jain et al. 2009), and they confirm that behind the mode frequency shift the active regions are not the only players. The other important feature is the anticorrelation over the minimum of solar cycle 23 for $\ell=0,2$. In these modes there are traces of the QBP signal. This might be the result of the different behaviors of the surface activity measure over the long extended minimum. While MWSI went down to unprecedented level until 2009, RF shows already an upward trend in February 2008. This might explain the higher degree of anticorrelation with RF than with MWSI.

\subsection{Azimuthal dependence of the correlation coefficient}

It is possible to investigate the latitudinal dependence of the correlation coefficient by using the single $m$-components of the modes provided by GONG data. In Fig. 9 we compare the degree of correlation between the zonal and sectoral components of dipole and quadrupole modes with MWSI and RF. The sectoral components seem to correlate better with both activity proxies over different phases of the solar cycle compared to the zonal modes. Previous works have shown that the central frequency of each multiplet is correlated best with the global activity measure, while the individual azimuthal frequencies are more sensitive to the latitudinal distribution of activity (Howe et al. 1999; Antia et al. 2001; Howe et al. 2002). Therefore the correlation analysis might suggest that the zonal modes are better correlated with resolved activity index that takes the latitudinal dependence of solar magnetic activity into account. To check this point, it will be important to extend the correlation analysis to the azimuthal components of intermediate to high-degree modes investigating the degree of correlation with resolved activity index.
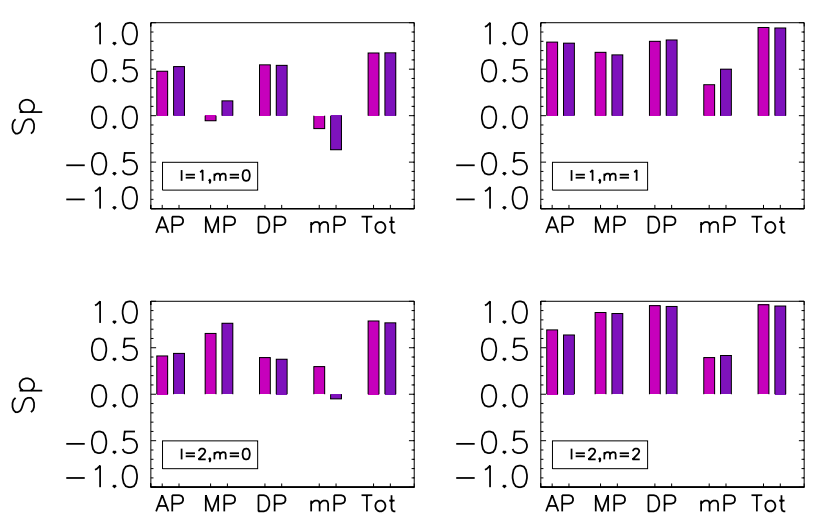

Fig. 9. The degree of correlation between $p$-mode frequency shifts of the azimuthal components of the modes and MWSI and RF. The bar colors identify MWSI (pink) and RF (purple).

\subsection{Conclusions}

Evidence of QBP has been found in the individual low $-\ell$ degree modes, as well as in their corresponding azimuthal $m$-components, at all levels of solar activity. The strength of the QBP signal seems to be modulated by the 11-year envelope, but the effect is more significant at low to middle latitudes compared to higher ones. This modulation might explain the coupling between the strength of the main global dynamo and the appearance of the QBP mainly over periods coinciding with solar maxima. When the solar cycle is extremely weak, the magnetic flux processed by the main dynamo is not strong enough to bring up the mid-term periodicity signal in those surface activity indices related to the toroidal component of the main dynamo.

The signatures of QBP have also been detected over the minimum phase of solar cycle 23 with $80 \%$ of confidence level. The likely persistent nature of the two-year periodicity in the seismic analysis must be investigated, but probably shows a permanent and periodic phenomenon that stands in the subsurface layers in addition to a pure presence of a magnetic field. The occurrence of the QBP signal over periods of solar maxima, as well as of solar minima, implies that the origin of the QBP is not coupled to the presence of active regions and therefore that the mechanism is different from the one that brings up to the surface the active regions. This finding agrees with previous results obtained by analyzing coronal-hole data (Vecchio \& Carbone 2008).

During periods of low activity, the QBP signal at low to middle latitudes is almost absent, while it appears at higher latitudes. This finding might imply that the signal strength increases with latitudes and also that the sources of excitation might cover all ranges in latitudes. This feature might be explained within the formalism of a second dynamo mechanism situated near the subsurface layer (Benevolenskaja 1998a,b).

The seismic investigation brings new insight into the features of solar magnetic activity that might be useful for improving dynamo models. To analyze the signature of the QBP, further investigation of the sectoral and zonal components of higher degree modes is strongly desired. We should also carry out a more detailed investigation of the latitudinal dependence of the signal strength over a solar cycle. The findings could be extremely useful for fully identifying the physical mechanism behind it, since at this early stage we cannot completely rule out the magnetic Rossby instability or other dynamo models that already take a further periodic component into account (Fluri \& Berdyugina 2004). Moreover, we would be able to check whether or not the 
Table 1. Spearmann correlation coefficient and significance between activity indices and low-degree $p$-mode frequency shift over different phases of solar activity.

\begin{tabular}{|c|c|c|c|c|c|c|c|c|c|c|}
\hline $\begin{array}{l}\text { Activity index } \\
\text { MWSI }\end{array}$ & $\begin{array}{c}\text { Mode of oscillation } \\
\ell \ell=0\end{array}$ & $\frac{\text { Data source }}{\text { BiSON }}$ & \multicolumn{2}{|c|}{$\mathrm{AP}$} & \multicolumn{2}{|c|}{ MP } & \multicolumn{2}{|c|}{ DP } & \multicolumn{2}{|c|}{$\mathrm{mP}$} \\
\hline MWSI & $\ell=0$ & $\begin{array}{c}\text { BiSON } \\
\text { GONG } \\
\text { VIRGO } \\
\text { GOLF }\end{array}$ & $\begin{array}{c}0.88 \\
0.80 \\
0.94 \\
-0.98\end{array}$ & $\begin{array}{l}3^{-5} \\
2^{-3} \\
4^{-7} \\
4^{-10}\end{array}$ & $\begin{array}{l}0.50 \\
0.43 \\
0.36 \\
0.27\end{array}$ & $\begin{array}{c}0.06 \\
0.09 \\
0.2 \\
0.3\end{array}$ & $\begin{array}{l}0.98 \\
0.87 \\
0.95 \\
0.97\end{array}$ & $\begin{array}{l}1^{-12} \\
1^{-5} \\
4^{-10} \\
1^{-11}\end{array}$ & $\begin{array}{c}-0.52 \\
0 \\
0.42 \\
0.36\end{array}$ & $\begin{array}{c}0.18 \\
0.1 \\
0.3 \\
0.4\end{array}$ \\
\hline & $\ell=1$ & $\begin{array}{c}\text { BiSON } \\
\text { GONG } \\
\text { VIRGO } \\
\text { GOLF }\end{array}$ & $\begin{array}{l}0.91 \\
0.97 \\
0.92 \\
0.92\end{array}$ & $\begin{array}{l}4^{-6} \\
1^{-7} \\
3^{-6} \\
4^{-6}\end{array}$ & $\begin{array}{c}0.52 \\
0.63 \\
0.40 \\
0.2\end{array}$ & $\begin{array}{c}0.04 \\
8^{-3} \\
0.13 \\
0.5\end{array}$ & $\begin{array}{l}0.94 \\
0.82 \\
0.94 \\
0.96\end{array}$ & $\begin{array}{l}2^{-9} \\
1^{-5} \\
3^{-9} \\
2^{-11}\end{array}$ & $\begin{array}{c}0.26 \\
-0.20 \\
0.67 \\
0.52\end{array}$ & $\begin{array}{c}0.5 \\
0.8 \\
0.08 \\
0.18\end{array}$ \\
\hline & $\ell=2$ & $\begin{array}{l}\text { BiSON } \\
\text { GONG } \\
\text { VIRGO } \\
\text { GOLF }\end{array}$ & $\begin{array}{l}0.92 \\
0.87 \\
0.77 \\
0.90\end{array}$ & $\begin{array}{l}4^{-6} \\
2^{-4} \\
1^{-3} \\
1^{-5}\end{array}$ & $\begin{array}{l}0.52 \\
0.60 \\
0.46 \\
0.48\end{array}$ & $\begin{array}{l}0.05 \\
0.01 \\
0.08 \\
0.07\end{array}$ & $\begin{array}{l}0.94 \\
0.95 \\
0.92 \\
0.90\end{array}$ & $\begin{array}{c}2^{-9} \\
2^{-10} \\
3^{-8} \\
1^{-7}\end{array}$ & $\begin{array}{l}0.26 \\
-0.2 \\
0.19 \\
0.36\end{array}$ & $\begin{array}{c}0.5 \\
0.8 \\
0.65 \\
0.38\end{array}$ \\
\hline $\mathrm{RF}$ & $\ell=0$ & $\begin{array}{l}\text { BiSON } \\
\text { GONG } \\
\text { VIRGO } \\
\text { GOLF }\end{array}$ & $\begin{array}{l}0.87 \\
0.80 \\
0.96 \\
0.99\end{array}$ & $\begin{array}{l}5^{-5} \\
2^{-3} \\
2^{-8} \\
6^{-12}\end{array}$ & $\begin{array}{l}0.71 \\
0.55 \\
0.94 \\
0.72\end{array}$ & $\begin{array}{c}3^{-3} \\
0.02 \\
1^{-7} \\
2^{-3}\end{array}$ & $\begin{array}{l}0.99 \\
0.83 \\
0.97 \\
0.98\end{array}$ & $\begin{array}{c}2^{-16} \\
1^{-5} \\
7^{-13} \\
1^{-14}\end{array}$ & $\begin{array}{c}-0.52 \\
0.40 \\
-0.24 \\
0.023\end{array}$ & $\begin{array}{c}0.2 \\
0.6 \\
0.57 \\
0.9\end{array}$ \\
\hline & $\ell=1$ & $\begin{array}{l}\text { BiSON } \\
\text { GONG } \\
\text { VIRGO } \\
\text { GOLF }\end{array}$ & $\begin{array}{l}0.97 \\
0.97 \\
0.95 \\
0.96\end{array}$ & $\begin{array}{l}4^{-9} \\
1^{-7} \\
2^{-6} \\
9^{-8}\end{array}$ & $\begin{array}{l}0.68 \\
0.74 \\
0.58 \\
0.67\end{array}$ & $\begin{array}{c}5^{-3} \\
9^{-4} \\
0.02 \\
6^{-4}\end{array}$ & $\begin{array}{l}0.95 \\
0.80 \\
0.93 \\
0.98\end{array}$ & $\begin{array}{c}2^{-10} \\
5^{-5} \\
4^{-11} \\
1^{-13}\end{array}$ & $\begin{array}{c}0.88 \\
0.93 \\
-0.14 \\
-0.05\end{array}$ & $\begin{array}{c}4^{-3} \\
4^{-11} \\
0.7 \\
0.9\end{array}$ \\
\hline & $\ell=2$ & $\begin{array}{l}\text { BiSON } \\
\text { GONG } \\
\text { VIRGO } \\
\text { GOLF }\end{array}$ & $\begin{array}{l}0.97 \\
0.87 \\
0.77 \\
0.91\end{array}$ & $\begin{array}{l}4^{-9} \\
2^{-4} \\
1^{-3} \\
5^{-6}\end{array}$ & $\begin{array}{l}0.89 \\
0.49 \\
0.83 \\
0.89\end{array}$ & $\begin{array}{c}7^{-6} \\
0.06 \\
1^{-4} \\
7^{-6}\end{array}$ & $\begin{array}{l}0.91 \\
0.94 \\
0.93 \\
0.93\end{array}$ & $\begin{array}{l}7^{-8} \\
1^{-9} \\
8^{-9} \\
1^{-8}\end{array}$ & $\begin{array}{l}-0.86 \\
-0.40 \\
-0.43 \\
-0.78\end{array}$ & $\begin{array}{c}6^{-3} \\
0.6 \\
0.3 \\
0.02\end{array}$ \\
\hline
\end{tabular}

Table 2. Same as Table 1 but for the azimuthal component of low-degree modes.

\begin{tabular}{lccccccccc}
\hline \hline Activity index & Mode of oscillation & \multicolumn{2}{c}{ AP } & \multicolumn{2}{c}{ MP } & \multicolumn{3}{c}{ DP } & \multicolumn{3}{c}{$\mathrm{mP}$} \\
\hline MWSI & $\ell=1, m=0$ & 0.48 & 0.1 & -0.05 & 0.8 & 0.55 & 0.03 & -0.14 & 0.7 \\
& $\ell=1, m=1$ & 0.79 & $1^{-3}$ & 0.68 & 0.01 & 0.80 & $1^{-4}$ & 0.33 & 0.34 \\
& $\ell=2, m=0$ & 0.41 & 0.16 & 0.65 & 0.01 & 0.39 & 0.13 & 0.30 & 0.13 \\
& $\ell=2, m=2$ & 0.69 & $9^{-3}$ & 0.88 & $7^{-5}$ & 0.95 & $1^{-8}$ & 0.39 & 0.3 \\
\hline RF & $\ell=1, m=0$ & 0.52 & 0.06 & 0.16 & 0.6 & 0.54 & 0.03 & -0.36 & 0.3 \\
& $\ell=1, m=1$ & 0.78 & $1^{-3}$ & 0.65 & 0.01 & 0.81 & $1^{-4}$ & 0.50 & 0.2 \\
& $\ell=2, m=0$ & 0.44 & 0.13 & 0.69 & 0.02 & 0.38 & 0.15 & -0.57 & 0.89 \\
& $\ell=2, m=2$ & 0.64 & 0.02 & 0.87 & $1^{-4}$ & 0.94 & $4^{-8}$ & 0.95 & 0.3 \\
\hline
\end{tabular}

periodicity differs in the two hemisphere, as already pointed out by Vecchio \& Carbone (2008).

The end of solar cycle 23, characterized by its long extended minimum, has re-opened interest in the particular dynamo mechanism that rules solar magnetic activity.

Acknowledgements. This work has been supported by the Swiss National Funding 200020-120114. This work utilizes data obtained by the Global Oscillation Network Group (GONG) program, managed by the National Solar Observatory, which is operated by AURA, Inc. under a cooperative agreement with the National Science Foundation. The data were acquired by instruments operated by the Big Bear Solar Observatory, High Altitude Observatory, Learmonth Solar Observatory, Udaipur Solar Observatory, Instituto de Astrofísica de Canarias, and Cerro Tololo Interamerican Observatory. Wavelet software was provided by $\mathrm{C}$. Torrence and G. Compo, and is available at URL: http://atoc.colorado.edu/research/wavelets/. We thank members of the BiSON team, both past and present, for their technical and analytical support. We also thank Y. Elsworth, W. J. Chaplin and A.-M. Broomahall for providing the data and useful comments to improve the manuscript. D.S. acknowledges the financial support from the Centre National d'Études Spatiales (CNES). R.S. is also grateful to S.Turck-Chièze and CEA/IRFU for providing the facilities required to continue her work. We also thank Dr. T. Zaqarashvili for useful discussion and suggestions for interpreting the results.

\section{References}

Akioka, M. 1987, Sol. Phys., 112, 313

Antia, H. M., Basu, S., Hill, F., et al. 2001, MNRAS, 327, 1029

Bai, T. 2003, ApJ, 585, 1114

Beckers, J. M., \& Brown, T. M. 1978, Oss. Mem. d. Oss. Astrofis. d. Arcetri, 106 Belmont, A. D., Darff, D., \& Ultad, M. S. 1996, J. Atmos. Sci., 23, 314

Benevolenskaja, E. 1995, Sol. Phys., 161, 1

Benevolenskaja, E. 1998a, ApJ, 509, L49

Benevolenskaja, E. 1998b, Sol. Phys., 181, 479

Broomhall, A. M., Chaplin, W. J., Elsworth, Y. P., Fletcher, S. T., \& New, R. 2009a, ApJ, 700, L162

Broomhall, A. M., Chaplin, W. J., Elsworth, Y. P., \& New, R. 2009b, MNRAS, 397,793

Broomhall, A. M., Chaplin, W. J., Elsworth, Y., \& Simoniello, R. 2011, MNRAS, 420,1405

Cadavid, A. C., Lawrence, J. K., McDonald, D. P., \& Ruzmaikin, A. 2005, Sol. Phys., 226, 359

Chaplin, W. J., Elsworth, Y. P., Howe, R., et al. 1995, MNRAS, 168, 1

Chaplin, W. J., Appourchaux, T., Elsworth, Y. P., \& Isaak, G. R. 2001, MNRAS, 324,910

Christensen-Dalsgaard, J. 2002, Rev. Mod. Phys., 74, 1073

Dziembowski, W. A., \& Goode, P. R. 2004, ApJ, 600, 464

Elsworth, Y. P., Howe, R., Isaak, G. R., McLeod, C. P., \& Miller, B. A. 1993, MNRAS, 265, 888 
Fletcher, S., Chaplin, W. J., Elsworth, Y., \& Roger, N. 2009, ApJ, 694, 144 Fletcher, S., Broomhall, A. M., Salabert, D., et al. 2010, ApJ, 718, L19 Fluri, D., \& Berdyugina, S. 2004, Sol. Phys., 224, 153

Fröhlich, C., Romero, J., Roth, H., et al. 1995, Sol. Phys., 162, 101

Fröhlich, C., Anderson, B., Appourchaux, T., et al. 1997, Sol. Phys., 170, 1

García, R. A., Turck-Chiéze, S., Boumier, P., et al. 2005, A\&A, 442, 385

Goode, P., \& Dziembowski, W. A. 2002, ESASP, 508, 156

Hindman, B. W., Haber, A. D., Toomre, J., \& Bogart, S. 2001, ESA SP, 464, 143

Howe, R., Komm, R., \& Hill, F. 1999, ApJ, 524, 1084

Howe, R., Komm, R., \& Hill, F. 2002, ApJ, 580, 1172

Jain, K., Tripathy, S., \& Hill, F. 2009, ApJ, 695, 1597

Jain, K., Tripathy, S., \& Hill, F. 2011, ApJ, 739, 6

Jiménez-Reyes, S. J., Corbard, T., Pallé, P., Roca Cortés, T., \& Tomczyk, S. 2001, A\&A, 379, 622

Jiménez, A., Jiménez-Reyes, S. J., \& García, R. A. 2005, ApJ, 623, 1215

Jiménez-Reyes, S. J., García, R., Chaplin, W. J., \& Korzennik, R. A. 2004, ApJ, 610,65

Jiménez-Reyes, S. J., Chaplin, W. J., \& Elsworth, Y. P. 2007, ApJ, 654, 1135

Knaack, R., Stenflo, J. O., \& Berdyugina, S. V. 2004, A\&A, 438, 1067

Komm, R. W., Howe, R., \& Hill, F. 2000, ApJ, 543, 472

Krivova, N., \& Solanki, S. 2002, A\&A, 394, 701

Lefebvre, S., Nighiem, P., \& Turck-Chièze, S. 2009, ApJ, 690, 1272

Li, K. J., Li, Q. X., Su, T. W., \& Gao, P. X. 2006, Sol. Phys., 239, 493

Mursula, K., Kieger, B., \& Vilppola, J. H. 2003, Sol. Phys., 212, 201

Nutto, C., Roth, M., Zhugzhda, Y., Bruls, J., \& von der Lühe, O. 2008, Sol. Phys.,

251,179
Pallé, P. L., Regulo, C., Roca-Cortés, T., \& Tomczyk, S. 1989, A\&A, 224, 253

Pallé, P. L., Régulo, C., Roca-Cortés, T., García, R. A., et al. 1999, A\&A, 341, 625

Penza, V., Pietropaolo, E., \& Livingston, W. 2006, A\&A, 355, 759

Salabert, D., \& Jiménez-Reyes, S. J. 2006, ApJ, 650, 451

Salabert, D., Fossat, E., Gelly, B., et al. 2004, A\&A, 413, 1135

Salabert, D., Chaplin, D., Elsworth, Y., New, R., \& Verner, G. 2007, A\&A, 463, 1181

Salabert, D., García, R. A., Pallé, P. L., \& Jiménez-Reyes, S. J. 2009, A\&A, 504, $1 \mathrm{~S}$

Salabert, D., Ballot, J., \& García, R. A. 2011, A\&A, 528, A25

Schou, J., Antia, H. M., Basu, S., et al. 1998, ApJ, 505, 390

Schunker, H., \& Cally, P. S. 2006, MNRAS, 372, 551

Simoniello, R., \& Salabert, D. García, R. A. 2009, ASPC, 416, 281

Simoniello, R., Finsterle, W., García, R. A., et al. 2010, A\&A, 516, A30

Tripathy, J., Jain, K., \& Hill, F. 2010, in Magnetic Coupling between the Interior and Atmosphere of the Sun, ed. S. S. Hasan, \& R. J. Rutten (Berlin, Heidelberg: Springer), Astrophys. Space Sci. Proc., 374

Ulrich, R. K., García, R. A., Robillot, J. M., et al. 2000, A\&A, 364, 799

Valdés-Galicia, J. F., \& Velasco, V. M. 2008, Adv. Space Res., 41, 291

Vecchio, A., \& Carbone, V. 2008, ApJ, 683, 536

Vecchio, A., Cauzzi, G., \& Reardon, K. 2009, A\&A, 494, 629

Woodard, M., \& Noyes, R. 1985, Nature, 318, 449

Zaqarashvili, T., Carbonell, M., Oliver, R., \& Ballester, J. L. 2010, ApJ, 724, 95

Zaqarashvili, T., Oliver, R., Ballester, J. L., et al. 2011, A\&A, 532, A139 